\title{
Stability of topological wall defects on spheres with $\boldsymbol{n}$-atic order
}

\author{
C. Saichand $\odot,{ }^{1}$ Jaya Kumar Alageshan, ${ }^{2}$ Arun Roy, ${ }^{1}$ and Yashodhan Hatwalne $e^{1, *}$ \\ ${ }^{1}$ Raman Research Institute, C.V. Raman Avenue, Bangalore 560 080, India \\ ${ }^{2}$ Indian Institute of Science, C.V. Raman Avenue, Bangalore 560 012, India
}

(Received 3 October 2019; accepted 27 April 2020; published 22 May 2020)

\begin{abstract}
Topological point defects on orientationally ordered spheres and on deformable fluid vesicles have been investigated, partly motivated by their potential applications in creating superatoms with directional bonds through functionalization of the "bald spots" created by topological point defects, thus paving the way for atomic chemistry at micron scales. We show that singular wall defects, topologically unstable "bald lines" in two dimensions, are stabilized near the order-disorder transition on a sphere. We attribute their stability to free-energetic considerations, which override those of topological stability.
\end{abstract}

DOI: 10.1103/PhysRevResearch.2.023215

\section{INTRODUCTION}

The remarkable interplay between the curvature of a surface and frustration of orientational order on it is strikingly demonstrated by the Poincaré-Hopf index theorem [1]. Stated informally, a hairy ball cannot be combed flat without creating at least one hair whorl; a singular isolated disclination (vortex) or isolated disclinations with total index (winding number) 2 . For vector (1-atic) order disclinations have integer indices, whereas for nematic (2-atic) order they are integer multiples of $1 / 2$. In a region surrounding a disclination (point defect in two dimensions), deformations in the orientation field are large enough to destroy orientational order. Disclinations are topological defects characterized by their index and have "molten" core regions of finite extent encompassing the disclination points.

The study of point disclinations on ordered spheres [2] and on deformable vesicles [3] is important for investigating the interplay between geometry, topology, and elasticity, and for its potential applications in materials science. It gained impetus from the proposal that disclination cores on spherical particles such as micron-scale colloidal particles coated with liquid crystals can be functionalized to create "superatoms" with directional bonds [4]. This opened up new possibilities such as self-assembly of superatoms by linking across functionalized groups (including biomolecules such as DNA) and the development of atomic chemistry at micron scales. Rigid spheres have been prepared by molecular coating of ordered tilted monolayer on metal nanospheres [5], leading to the antipodal configuration of a source-sink pair of disclinations of index 1 each. These divalent superatoms spontaneously form long

\footnotetext{
*Corresponding author: yhat@ rri.res.in

Published by the American Physical Society under the terms of the Creative Commons Attribution 4.0 International license. Further distribution of this work must maintain attribution to the author(s) and the published article's title, journal citation, and DOI.
}

one-dimensional chains. Thin nematic shells consisting of a nematic drop containing a smaller aqueous drop have been obtained in double emulsions [6]. These can be engineered to imitate $s p$-, $s p^{2}$-, and $s p^{3}$ geometries of carbon bonds. Deformable vesicles with orientational order can form facets. These fascinating possibilities have led to rapid advances in theoretical and experimental studies [7-11].

In this paper we address the energetics and stability of topological wall defects (line defects in two dimensions) on spherical fluid membranes with $n$-atic orientational order. Singular wall defects in two- and three-dimensional ordered systems are topologically unstable because they can be made to disappear by making local changes in the orientational order $[12,13]$. In three dimensions, removal of disclinations lines with index 1 via "escape" of the nematic director in the third dimension [12-16] is well known. However, close to nematic-smectic transition the bend elastic constant diverges, the escape configuration has a larger free energy than that of the line disclination of index 1 , and the disclination line is stabilized.

We show that singular wall defects can be stabilized on a sphere because of its Gaussian (intrinsic) curvature, and not because of boundary conditions, externally imposed fields, or divergences in certain elastic constants. They are stable close to the order-disorder transition, over a finite range of a dimensionless parameter $\eta$. The parameter $\eta$ is the ratio of basic free energy scales corresponding to the destruction of order in the defect cores to that of the elastic deformation outside the core. Our results on vector and nematic orders are summarized in Figs. 1-5. The extension of these results to $n$-atic order is straightforward. Remarkably, we find that for $n$-atic order, the lowest elastic free-energy configuration has $2 n$ walls of index $1 / n$ each, located such that the integral of Gaussian curvature of the sphere between any two successive walls is $2 \pi / n$. The one-dimensional closed loop walls that we consider have an unusual feature-each loop is characterized by a continuous disclination density along its length, with a quantized index assigned to the entire loop [17]. To the best of our knowledge, such defects have not been discussed in condensed matter systems. 


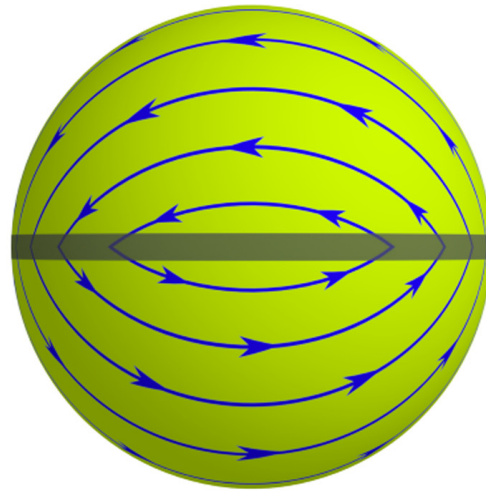

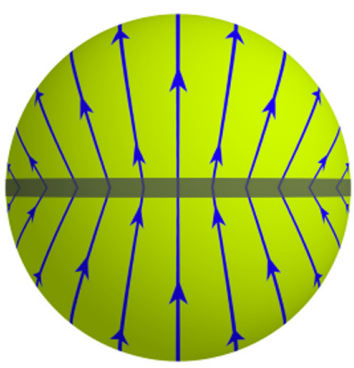

(b) (a)

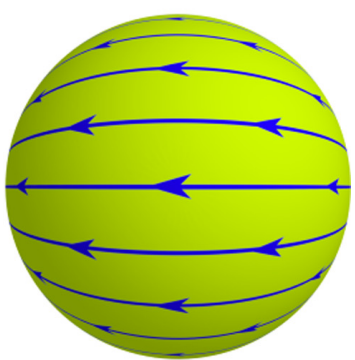

(c)
FIG. 1. Equatorial wall: (a) Side $(\theta=\pi / 2, \phi=\pi / 2)$ view of the index 2, equatorial wall. The directed lines are the streamlines of the vector field. The shaded region represents the disordered core, within which vector order is completely destroyed, and cannot be assigned a direction. The full field, including that shown within the core region, corresponds to a wall with zero core size. Rounding off the slope singularity of the field at the equator, the wall defect of zero core size transforms into the antipodal configuration of a pair of index 1 point disclinations. (b) Front view $(\theta=\pi / 2, \phi=0)$. (c) Top view, showing that the polar regions are free of point disclinations.

\section{THE MODEL}

On curved orientationally ordered surfaces the free energy takes the form of Ginzburg-Landau theory for superconductivity [3]. Apart from the metric tensor, the complex order parameter is coupled to the shape through a gauge field arising from the covariant derivative of the order parameter. For $n$-atic order a disclination of index $(p / n)$ where $p$ is an integer, is a singular point around which the phase angle of the order parameter changes by $2 \pi(p / n)$. In superconductors the total vorticity is determined by an external magnetic field, whereas for a surface the signed total index of the disclinations is fixed by its topology. The free energy density of orientationally ordered surfaces can be cast as

$$
f_{\mathrm{GL}}=\frac{r}{2}|\psi|^{2}+\frac{u}{4}|\psi|^{4}+\frac{k}{2}|(\boldsymbol{\partial}-i \boldsymbol{A}) \psi|^{2},
$$

where $r=r_{0}\left(T-T_{c}\right), T$ and $T_{c}$ are the temperature, and the critical temperature, respectively, $u$ is assumed to be temperature independent, and $\psi=\psi(\underline{\boldsymbol{\sigma}})=|\psi(\underline{\boldsymbol{\sigma}})| e^{i \alpha(\underline{\boldsymbol{\sigma}})}$ is the complex order parameter. For $n$-atic order the angle $\alpha$ is measured modulo $2 \pi / n$ with respect to a local orthonormal frame $\left[\hat{\boldsymbol{e}}_{1}(\underline{\boldsymbol{\sigma}}), \hat{\boldsymbol{e}}_{2}(\underline{\sigma})\right]$ on the surface, where $\underline{\sigma}=\left(\sigma^{1}, \sigma^{2}\right)$ represent the internal coordinates parametrizing the surface. The term with coefficient $k$ describes the elastic free energy of deformations within the "one-constant" approximation [12-14]. In this term $\partial$ represents the flat-space gradient operator, and $\boldsymbol{A}(\underline{\sigma})$ is the local gauge field (the spin connection) that corrects the flat-space gradient by accounting for membrane curvature. The components of the spin connection are given by $A_{\mu}(\underline{\boldsymbol{\sigma}})=\hat{\boldsymbol{e}}_{1}(\underline{\boldsymbol{\sigma}}) \cdot \partial_{\mu} \hat{\boldsymbol{e}}_{2}(\underline{\boldsymbol{\sigma}})$, where $\mu \in\left(\sigma^{1}, \sigma^{2}\right)$. In the term with coefficient $k$, complex conjugates are contracted using the metric tensor. The free energy $F_{\mathrm{GL}}=\int f_{\mathrm{GL}} d \mathcal{A}$, where the area element $d \mathcal{A}=\sqrt{g} \mathrm{~d} \sigma^{1} \mathrm{~d} \sigma^{2}$, and $g$ is the determinant of the metric tensor $g_{\mu \nu}$. The condensation free energy density (the free energy cost per unit area for destruction of orientational order) $f_{\mathrm{C}}=r^{2} /(4 u)$. It plays an important role in determining the core size of topological defects [13]. The elastic part of the free energy density (1) is

$$
f_{\mathrm{el}}=\frac{1}{2} K_{\alpha}(\partial \alpha-\boldsymbol{A})^{2}=\frac{1}{2} K_{\alpha}\left(\partial_{\mu} \alpha-A_{\mu}\right)\left(\partial^{\mu} \alpha-A^{\mu}\right),
$$

where $K_{\alpha}=k|\psi|^{2}$ in the mean field approximation. Minimization of the elastic free energy $F_{\mathrm{el}}=\int f_{\mathrm{el}} \mathrm{d} \mathcal{A}$ leads to the Euler-Lagrange equation of equilibrium $\left(\delta F_{\mathrm{el}} / \delta \alpha\right)=-K_{\alpha} \nabla$. $(\partial \alpha-\boldsymbol{A})=0$, where the operator $\nabla \cdot$ denotes the covariant divergence. For flat surfaces the gauge field $\boldsymbol{A}=0$, and $F_{\mathrm{el}}$ reduces to the elastic free energy density of the continuum $x y$ model. The Airy stress function $\chi$ defined by $\partial^{\mu} \alpha-A^{\mu}=$ $\gamma^{\mu \nu} \partial_{\nu} \chi$, with the unit antisymmetric tensor density $\gamma^{\mu \nu}=$ $\epsilon^{\mu \nu} / \sqrt{g}$, identically satisfies $\left(\delta F_{\mathrm{el}} / \delta \alpha\right)=0$. However, $\chi$ has to obey the compatibility condition [18-20]

$$
\nabla^{2} \chi(\underline{\sigma})=K(\underline{\sigma})-\mathscr{S}(\underline{\sigma}) .
$$

Here, $\nabla^{2}$ is the covariant Laplacian operator, $K(\sigma)$ is the Gaussian curvature, and $\mathscr{S}(\underline{\sigma})$ is the disclination density [21]. In terms of the stress function $\chi$, the elastic free energy density (2) can be written as

$$
f_{\mathrm{el}}=\frac{1}{2} K_{\alpha}(\partial \chi)^{2}=\frac{1}{2} K_{\alpha}\left(\partial_{\mu} \chi\right)\left(\partial^{\mu} \chi\right) .
$$

\section{DEFECT CONFIGURATIONS}

With this background we discuss the procedure followed in calculating the free energies of point and wall defects on a sphere of radius $R$. For vector order the free energy of the antipodal configuration of (index 1) point disclinations has been obtained for small core sizes $r_{\mathrm{c}}$, where the dimensionless cutoff $\zeta=r_{\mathrm{c}} / R \ll 1$ [2]. A larger core reduces the elastic free energy $F_{\mathrm{el}}$ but increases the condensation free energy. Making the simplifying assumption that order is destroyed over the entire core region, the condensation free energy $F_{\mathrm{C}}=\int f_{\mathrm{C}} d \mathcal{A}$, where the integral is over the core region [13]. In investigating the energetics and stability of point as well as wall defects for $n$-atic order the determination of optimal core sizes, without the restriction $\zeta \ll 1$, is crucial. In what follows, we minimize the dimensionless total free energies $F_{\mathrm{T}}=\left(F_{\mathrm{el}}+F_{\mathrm{C}}\right) / K_{\alpha}$ with respect to $\zeta$ to obtain the optimal core size. We focus primarily on vector and nematic orders, and discuss the antipodal-, equatorial wall-, two-wall-, tetrahedral-, and four-wall configurations. We find that these configurations are ground states in different ranges of $\eta=f_{\mathrm{C}} R^{2} / K_{\alpha}$. To compare their total free energies and ranges of stability, it is convenient to choose the dimensionless condensation energy $4 \pi \eta$ corresponding to 
the destruction of order over the entire sphere as the common reference of free energy.

\section{A. The antipodal configuration}

Let us consider a unit tangent vector field $\hat{\boldsymbol{m}}(\theta, \phi)=$ $\cos \alpha(\theta, \phi) \hat{\boldsymbol{e}}_{\theta}+\sin \alpha(\theta, \phi) \hat{\boldsymbol{e}}_{\phi}$ on a sphere, where we use spherical polar coordinates. For a sphere of radius $R$, the Gaussian curvature $K=1 / R^{2}, A_{\theta}=0, A_{\phi}=-\cos \theta$, and the components of the metric tensor are $g_{\theta \theta}=R^{2}, g_{\phi \phi}=$ $R^{2} \sin ^{2} \theta, g_{\theta \phi}=g_{\phi \theta}=0$.

For the simplest case that describes the antipodal configuration, $\alpha=0$ (streamlines of $\hat{\boldsymbol{m}}$ follow longitudes on the sphere), with two point disclinations of index 1 each at the poles. The elastic free energy of the vectorial texture outside the core region is

$$
F_{\text {el }}^{(\text {ap })}=2 \pi K_{\alpha}[\log \cot (\zeta / 2)-\cos \zeta],
$$

where the superscript (ap) stands for antipodal point disclinations, and the dimensionless cutoff $\zeta=r_{\mathrm{c}} / R$. For small $r_{\mathrm{c}}$ this result reduces to that of [2]. We note that $F_{\mathrm{el}}^{\text {(ap) }}$ depends solely on $\zeta$ and diverges logarithmically as $\zeta \rightarrow 0$. The condensation free energy is

$$
F_{\mathrm{C}}^{(\mathrm{ap})}=4 \pi R^{2} f_{\mathrm{C}}(1-\cos \zeta) .
$$

For antipodal points the optimum core size is

$$
\zeta^{(\text {ap })}=2 \arctan [p(\eta) / q(\eta)],
$$

where $p(\eta)=[1+2 \eta-\sqrt{2 \eta(1+2 \eta)}]^{1 / 2}$, and $q(\eta)=[1+$ $2 \eta+\sqrt{2 \eta(1+2 \eta)}]^{1 / 2}$. The minimized total (elastic + condensation) dimensionless free energy of the antipodal configuration in units of $K_{\alpha}$ is

$$
\mathcal{F}^{\text {(ap) }}=2 \pi[2 \eta-\sqrt{2} h(\eta)+\operatorname{arctanh}(\sqrt{2} \eta / h(\eta))]-4 \pi \eta,
$$

where $h(\eta)=\sqrt{\eta(1+2 \eta)}$, and we have taken the dimensionless condensation free energy $4 \pi \eta$ for destruction of order over the entire sphere as the reference. The expressions for the optimized core size $\zeta^{(\mathrm{ap})}$ and the minimized total free energy $\mathcal{F}^{\text {(ap) }}$ of the antipodal configuration are plotted in Figs. 4 and 5 as functions of $\eta$. Within the mean field theory, $\eta \sim\left(T_{c}-T\right)$.

\section{B. The equatorial wall configuration}

We now consider a singular equatorial wall defect (at $\theta=$ $\pi / 2$ ), defined by the disclination density

$$
\mathscr{S}^{(\mathrm{ew})}=\frac{S_{0}}{2 \pi \sqrt{g}} \delta\left(\theta-\frac{\pi}{2}\right),
$$

where the superscript (ew) stands for equatorial wall. Thus $\int_{0}^{2 \pi} d \phi \int_{0}^{\pi} \mathscr{S}^{(\mathrm{ew})} \sqrt{g} d \theta=S_{0}$, where $S_{0}$ is as yet undetermined. Given this disclination density, we seek a solution to the compatibility condition (3) subject to boundary conditions. The boundary conditions, discussed below, ensure that there are no point disclinations at the poles (coordinate singularities) and that $S_{0}=4 \pi$, vindicating the Poincaré-Hopf index theorem [22].

The general solution to the compatibility condition (3) is

$$
\begin{aligned}
\chi(\theta)= & -a_{1} \log \tan (\theta / 2)-\log \sin \theta+\left[S_{0} /(2 \pi)\right] \\
& \times[\Theta(\theta-(\pi / 2))-1] \log \cot (\theta / 2)+a_{2},
\end{aligned}
$$

where the symbol $\Theta$ is the Heaviside theta, and $a_{1}, a_{2}$ are constants. Setting $a_{2}=0$, we exploit the symmetry $\chi(\theta)=$ $\chi(\pi-\theta)$ to obtain $a_{1}=-S_{0} /(4 \pi)$. Note that $S_{0}$ is as yet undetermined. To ensure that there are no point disclinations of index 1 each at the north and south poles, we investigate the behavior of $\partial_{\theta} \chi(\theta)$ at the poles. We note that $\lim _{\theta \rightarrow 0} \partial_{\theta} \chi(\theta)$ as well as $\lim _{\theta \rightarrow \pi} \partial_{\theta} \chi$ go to infinity unless $S_{0}=4 \pi$. With $S_{0}=4 \pi$, both these limits go to zero. Setting $a_{1}=-S_{0} /(4 \pi)=-1$ guarantees that there are no point disclinations at the north and south poles and yields the solution to the compatibility condition for the equatorial wall,

$$
\chi^{(\mathrm{ew})}=2\left[\Theta\left(\theta-\frac{\pi}{2}\right) \log \cot \frac{\theta}{2}-\log \left(\sqrt{2} \cos \frac{\theta}{2}\right)\right] .
$$

We have thus constructed a wall defect with index 2 , as demanded by the Poincaré-Hopf theorem extended to nonisolated zeros. The Heaviside $\Theta$ in (11) has important consequences for the stability of wall defects, as discussed below. In terms of $\alpha$, measured in the local orthonormal frame $\left[\hat{\boldsymbol{e}}_{\theta}(\theta, \phi), \hat{\boldsymbol{e}}_{\phi}(\theta, \phi)\right]$, the solution (11) is particularly simple (Fig. 1): $\alpha^{(\mathrm{ew})}=-\phi$, if $\theta<\pi / 2 ; \alpha^{(\mathrm{ew})}=\phi$, if $\pi>$ $\theta>\pi / 2$ [23]. Substituting for (11) in (4), the elastic free energy of the equatorial wall is

$$
F_{\mathrm{el}}^{(\mathrm{ew})}=2 \pi K_{\alpha}\{\sin \zeta-4 \log \sin [(\zeta / 2)+(\pi / 4)]-1\} .
$$

We note that the term with the Heaviside $\Theta$ in (11), when substituted in $f_{\mathrm{el}}[\chi](4)$, leads to a term involving $(\delta[\theta-$ $(\pi / 2)])^{2}$ in the integrand. With $\zeta=0$ at or within the limits of integration, the integral is, strictly speaking, undefined. However it diverges as $1 / \zeta$ [24], in contrast to the logarithmic divergence encountered in the case of antipodal point disclinations. We therefore expect the index 2 wall to become unstable as $\zeta$ approaches molecular size, within the coarse-grained elasticity theory that we have used. This is borne out by the minimization of the total free energy discussed below.

The condensation free energy is given by

$$
F_{\mathrm{C}}^{(\mathrm{ew})}=4 \pi R^{2} f_{\mathrm{C}} \sin \zeta .
$$

The core size $\zeta^{(\mathrm{ew})}$ that minimizes the total free energy $\mathcal{F}^{\text {(ew) }}=F_{\mathrm{T}}^{\text {(ew) }}-4 \pi \eta$ is

$$
\zeta^{(\mathrm{ew})}=\arctan \frac{1-2 \eta}{2 \sqrt{2 \eta}}
$$

and is plotted in Fig. 4 as a function of $\eta$. We find that $\zeta^{(\mathrm{ew})}=0$ at $\eta=1 / 2$. Moreover, it crosses zero and is negative above $\eta=1 / 2$, which is unphysical. Thus, the singular equatorial wall is stable only for $0<\eta<1 / 2$. The minimized dimensionless total free energy of the equatorial wall (Fig. 5) with reference to the dimensionless condensation energy $4 \pi \eta$ is

$$
\mathcal{F}^{\text {(ew) }}=4 \pi[\log (1+2 \eta)-2 \eta],
$$

where we recall that $\eta=\left(f_{\mathrm{C}} R^{2}\right) / K_{\alpha} \sim\left|T-T_{c}\right|$. As in the case of antipodal point disclinations, $\mathcal{F}^{\text {(ew) }}$ depends only on $\eta$.

\section{The two-wall configuration}

The equatorial wall is not necessarily the minimum energy configuration (ground state for vectorial order) over its entire 


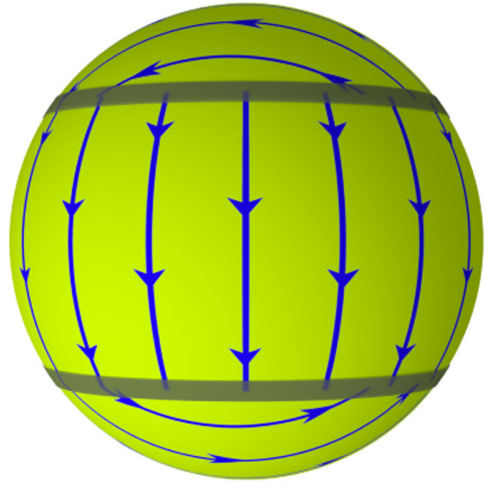

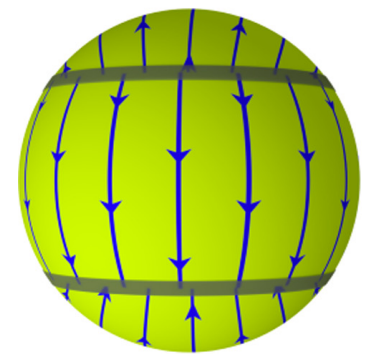

(b) (a)

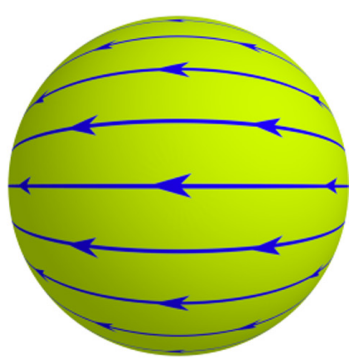

(c)
FIG. 2. Two-wall configuration: (a) Side view. The vector field between the two index 1 walls follows the longitudes. The two-wall configuration degenerates to the antipodal configuration by smoothly sliding the walls towards the respective poles. (b) Front view. (c) Top view.

range of stability. For vector as well as nematic order it can split into two walls with index 1 each placed at $\theta=\omega_{1}^{(2 \mathrm{w})}$, and $\omega_{2}^{(2 \mathrm{w})}=\pi-\omega_{1}^{(2 \mathrm{w})}$, where $\omega_{1}^{(2 \mathrm{w})}<\pi / 2$. In terms of the $\alpha$ field, the solution to the compatibility condition (3) for the two-wall configuration is $\alpha^{(2 \mathrm{w})}=(-\phi, 0, \phi)$ in the order of increasing $\theta$ (Fig. 2). Following the same procedure as used above for the equatorial wall, we find that the numerically minimized total free energy of the two-wall configuration determines the wall locations $\omega_{1}^{(2 \mathrm{w})}(\eta)$ and $\omega_{2}^{(2 \mathrm{w})}(\eta)$. The twowall configuration is stable only for $0<\eta<0.17$. The angle $\omega_{1}^{(2 \mathrm{w})}(\eta)$ is a monotonic increasing function with $\omega_{1}^{(2 \mathrm{w})}(\eta=$ $0)=\pi / 4$ (corresponding to destruction of order over the entire sphere), and the angle corresponding to zero cutoff can be analytically calculated to be $\omega_{1}^{(2 \mathrm{w})}(\eta=0.17)=\pi / 3$. At the limit of stability $(\eta=0.17)$ the total (integrated) Gaussian curvature of the spherical region between the two walls $K_{\mathrm{T}}=2 \pi$, leaving total Gaussian curvatures of $\pi$ each for the polar caps. We notice a similar trend for the division of total Gaussian curvature between successive walls in the index 1/2, four-wall configuration for nematic order (see below).

\section{The tetrahedral configuration}

We now discuss the tetrahedral configuration of point disclinations of index $1 / 2$, followed by the four-wall configuration (of index $1 / 2$ each) for nematic order. It is known [2] that for small core sizes the ground state has four disclinations arranged on the vertices of a tetrahedron inscribed in the sphere. For the tetrahedral configuration, we minimize the

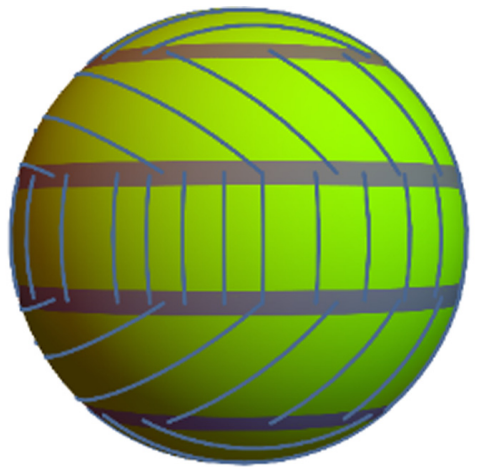

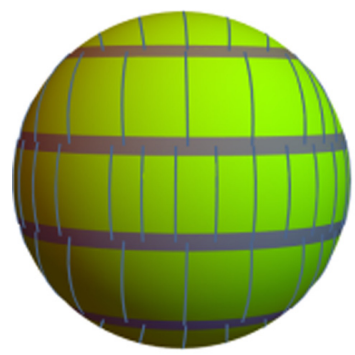

(b) (a)

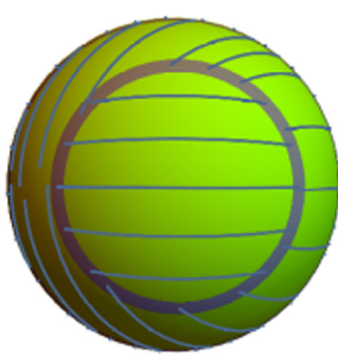

(c)
FIG. 3. Four-wall configuration (for nematic order): (a) Side view. (b) $\operatorname{Back}(\theta \simeq \pi / 2, \phi \simeq 3 \pi / 2)$ view. (c) Top view. Each wall in this configuration has the index $1 / 2$, with uniform linear disclination density. Therefore the change in the angle per unit length across each wall is small as compared to that for index 1 (and 2) walls. This should be borne in mind, particularly while viewing the back view (b).

total free energy numerically, using equal cutoffs along the $\theta$ and $\phi$ directions to obtain the optimized core size (Fig. 4) and the total free energy (Fig. 5). Above $\zeta \simeq 0.96$ (below $\eta(\zeta=0.96) \simeq 0.05$ ), cores of neighboring disclinations overlap each other at nonzero $\eta$, and an adaptation of the approach of Ref. [25] that treats the disorder to order transition is better suited to address this problem. In this paper we do not pursue this approach.

\section{E. The four-wall configuration}

The four-wall configuration (Fig. 3) has two walls located at $\theta=\left(\omega_{1}^{(4 \mathrm{w})}, \omega_{2}^{(4 \mathrm{w})}>\omega_{1}^{(4 \mathrm{w})}\right)$ on the upper hemisphere. The other two symmetry related walls are located in the lower hemisphere, with the convention $\omega_{3}^{(4 \mathrm{w})}<\omega_{4}^{(4 \mathrm{w})}$. The solution to the compatibility condition gives $\alpha^{(4 \mathrm{w})}=$ $(-\phi,-\phi / 2,0, \phi / 2, \phi)$ in the five regions arranged in the order of increasing $\theta$. We find that this configuration is stable only for $0<\eta<0.05$. In minimizing the total free energy of the pairs of walls we need to use two core sizes, $\zeta_{1}^{(4 \mathrm{w})}$ and $\zeta_{2}^{(4 \mathrm{w})}$, for the walls at $\omega_{1}^{(4 \mathrm{w})}$ and $\omega_{2}^{(4 \mathrm{w})}$, respectively. The angles $\omega_{1}^{(4 \mathrm{w})}$ and $\omega_{2}^{(4 \mathrm{w})}$ are weakly monotonic increasing functions of $\eta$, with $\omega_{1}^{(4 \mathrm{w})}(\eta=0.05)=2 \arctan (1 / \sqrt{7})(\simeq 0.72)$, and $\omega_{2}^{(4 \mathrm{w})}(\eta=0.05)=2 \arctan (\sqrt{(3 / 5})(\simeq 1.32)$. We notice that putting the exact values as limits of integration, we get $K_{\mathrm{T}}=$ $\pi$, indicating a systematic division of total Gaussian curvature between neighboring walls. In what follows we trace this trend 


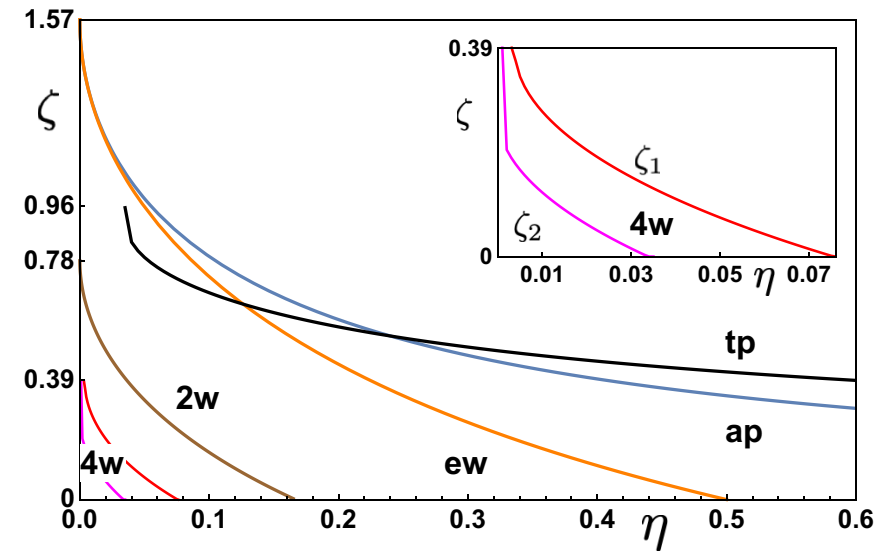

FIG. 4. Optimized core sizes: The symbols ap and tp represent the antipodal (index 1 each) and tetrahedral (index $1 / 2$ each) configurations of point disclinations; ew, $2 \mathrm{w}$, and $4 \mathrm{w}$ refer to the equatorial (index 2), two-wall (index 1 each), and four-wall (index 1/2 each) configurations, respectively. The inset depicts the two core sizes required to minimize the total free energy for the $4 \mathrm{w}$ configuration. Order is completely destroyed at $\zeta=\{\pi / 8, \pi / 4, \pi / 2, \pi / 2\}$ for the $\{4 \mathrm{w}, 2 \mathrm{w}, \mathrm{ew}, \mathrm{ap}\}$ configurations respectively. Above $\zeta=$ $(1 / 2) \arccos (-1 / 3) \simeq 0.96$, cores of neighboring disclinations of the tp configuration overlap each other (see the text).

to the elastic part of the free energy and generalize it to $n$-atic order.

We find that configurations involving intersecting walls (for example, longitudinal walls along $\phi=0$ and $\phi=\pi / 2$, vectorial order) have higher free energies than the configurations discussed above. This is because of the larger repulsive interaction energy near their intersection points, where the walls are close.

\section{F. Wall defects: Feasibility of observation}

Wall defects are stable in the range $0<\eta<0.5$. We use mean field theory to examine the extent to which wall defects are realizable in experiments. This involves estimating the radius $R$, the thickness $h$, and the temperature range $\Delta T=$ $T_{c}-T$ over which stable wall defects are observable. We recall that the scale of $\eta$ in Figs. 4 and 5 is linear in $\Delta T$.

For the sake of concreteness we consider the experiments of Ref. [5], where molecules of the self assembled monolayer (thickness $h$ of order molecular length) on the sphere are tilted with respect to the local normal to the sphere. The projection of tilted molecules onto the local tangent plane to the sphere imparts vectorial order to it. Within mean field theory the correlation length diverges as $\xi=\xi_{0}\left(\Delta T / T_{c}\right)^{-1 / 2}$. The bare correlation length $\xi_{0}$ is of order $2 \mathrm{~nm}$ (molecular dimensions), and $K_{\alpha} \simeq k_{\mathrm{B}} \Delta T$, leading to $f_{\mathrm{C}} \simeq k_{\mathrm{B}} \Delta T / \xi^{2}$. Substituting for $\xi$ in $\eta$, we get $\Delta T \simeq T_{c}\left(\xi_{0} / R\right)^{2} \eta$. For $T_{c} \simeq 300 \mathrm{~K}$, spheres with $R=5 \mathrm{~nm}$, and $R=10 \mathrm{~nm}$ (used in [5]), $\eta=0.1$ corresponds to $\Delta T \simeq 4.8 \mathrm{~K}$ and $\Delta T \simeq 1.2 \mathrm{~K}$, respectively, thus establishing the temperature scale. For $R \simeq 35 \mathrm{~nm}, \eta \simeq 0.1$ corresponds to $\Delta T \simeq 0.1 \mathrm{~K}$. For $R=35 \mathrm{~nm}$ the equatorial wall is stable between $\eta \simeq 0.16(\equiv \Delta T=0.16 \mathrm{~K})$ and $\eta=$ $0.5(\equiv \Delta T=0.5 \mathrm{~K})$; it is likely to be the simplest one to

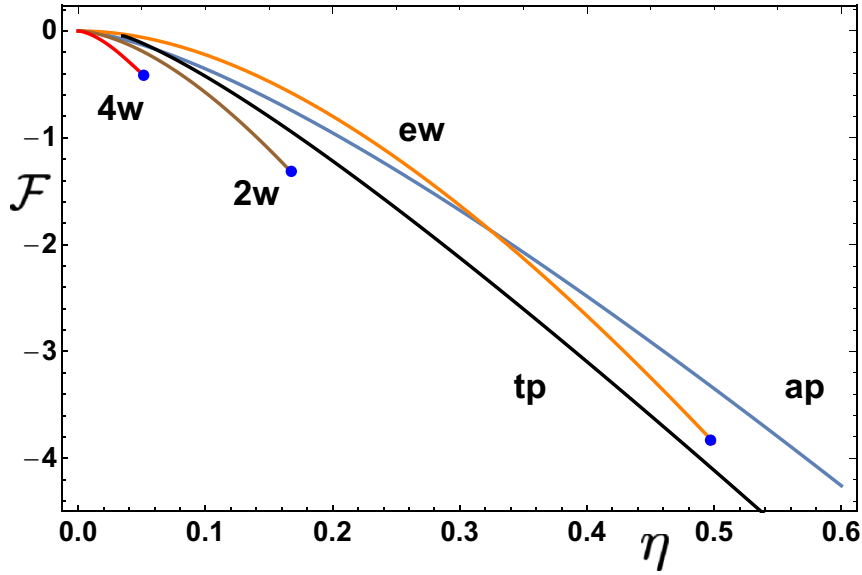

FIG. 5. Minimized free energy $\mathcal{F}=F_{\mathrm{T}}-4 \pi \eta$ : We set the reference of the total free energy to the condensation energy $4 \pi \eta$ of the entire sphere. The dots indicate the $\eta$ values beyond which wall defects are unstable $[\zeta(\eta)=0]$, see Fig. 4 .

observe. Evidently, for large core sizes, point as well as wall defects will not have a "valence" $=1$, as is the case for the antipodal disclinations of Ref. [5]. However, nanoparticles with small radii of order $35 \mathrm{~nm}$ are faceted [26] and cannot be reliably approximated as spheres. Thus the observation of wall defects (as against antipodal point disclinations) using nanoparticles may not yet be possible using available experimental techniques.

\section{G. Wall defects: Division of total Gaussian curvature}

We now generalize, and make precise our remarks on the systematics of the division of total Gaussian curvature between neighboring walls. For core sizes $\zeta \rightarrow 0$ the condensation energy is negligibly small, clearly implicating the elastic free energy as the root cause of this phenomenon. By minimizing the elastic free energy, we find that for $n$-atic order on spheres the lowest elastic free energy configuration has $2 n$ walls of index $1 / n$ each, located such that the integrated Gaussian curvature between any two successive walls

$$
K_{\mathrm{T}}(i, i+1)=2 \pi \int_{\omega_{i}}^{\omega_{i+1}} K \sqrt{g} d \theta=2 \pi / n,
$$

where $i=\{1,2, \ldots, 2 n-1\}$ labels the walls in the order of increasing $\theta$.

To derive this result, let us consider $n$-atic order on a sphere with $2 n$ walls of strength $1 / n$ each and indicate the positions of symmetry related pairs of walls by $\left(\omega_{k}, \omega_{-k}=\right.$ $\left.\pi-\omega_{k}\right)$, where $k=1,2, \ldots, n$. Our aim is to minimize the elastic free energy of such configurations with respect to the angular positions of the symmetry-related pairs of walls. The disclination density of this configuration is

$$
\mathscr{S}(\theta)=\frac{1}{n \sqrt{g}} \sum_{k=1}^{n}\left[\delta\left(\theta-\omega_{k}\right)+\delta\left(\theta-\omega_{-k}\right)\right] .
$$

To evaluate the elastic free energy of the configuration we use the Coulomb gas form of the elastic free energy. The elastic 
energy $F_{\text {el }}$ can be written as [7]

$$
F_{\mathrm{el}}=-\frac{K_{\alpha}}{2} \iint \rho(\theta, \phi) G\left(\theta, \phi ; \theta^{\prime}, \phi^{\prime}\right) \rho\left(\theta^{\prime}, \phi^{\prime}\right) d \mathcal{A} d \mathcal{A}^{\prime},
$$

where $\rho(\theta, \phi)=K(\theta, \phi)-\mathscr{S}(\theta, \phi)$. The Green's function $G\left(\underline{\sigma}, \underline{\sigma}^{\prime}\right)$ for a spherical surface is

$$
G\left(\theta, \phi ; \theta^{\prime}, \phi^{\prime}\right)=\frac{1}{4 \pi} \log \left[\frac{1-C_{\beta}\left(\theta, \phi ; \theta^{\prime}, \phi^{\prime}\right)}{2}\right],
$$

where

$$
C_{\beta}\left(\theta, \phi ; \theta^{\prime}, \phi^{\prime}\right)=\cos \theta \cos \theta^{\prime}+\sin \theta \sin \theta^{\prime} \cos \left(\phi-\phi^{\prime}\right) .
$$

Given the azimuthal symmetry of the configuration (no $\phi$ dependence), the Green's function satisfies the Laplace equation

$$
\sin \theta \partial_{\theta}^{2} G\left(\theta ; \theta^{\prime}\right)+\cos \theta \partial_{\theta} G\left(\theta ; \theta^{\prime}\right)+\frac{\sin \theta}{4 \pi}=\frac{\delta\left(\theta, \theta^{\prime}\right)}{2 \pi},
$$

with the following conditions: (i) $G\left(\theta ; \theta^{\prime}\right)=G\left(\theta^{\prime} ; \theta\right)$, and (ii) $G\left(\pi-\theta ; \pi-\theta^{\prime}\right)=G\left(\theta ; \theta^{\prime}\right)$. The solution of the above equation is

$$
\begin{aligned}
G\left(\theta ; \theta^{\prime}\right)= & \frac{1}{8 \pi}\left[\log \left(\sin \theta \sin \theta^{\prime}\right)+\left(\Theta\left(\theta^{\prime}-\theta\right)\right.\right. \\
& \left.\left.-\Theta\left(\theta-\theta^{\prime}\right)\right) \log \left(\cot \frac{\theta}{2} \tan \frac{\theta^{\prime}}{2}\right)\right],
\end{aligned}
$$

where the Heaviside theta is defined as $\Theta(x)=1$ if $x>0$, and $\Theta(x)=0$ if $x<0$.

The elastic free energy (18) can be written as $F_{\mathrm{el}}=$ $-(1 / 2) K_{\alpha}\left(F_{K K}-2 F_{K \mathscr{S}}+F_{\mathscr{S} \mathscr{S}}\right)$, where

$$
\begin{aligned}
F_{K K} & =\iint K G\left(\theta ; \theta^{\prime}\right) K d \mathcal{A} d \mathcal{A}^{\prime}, \\
F_{K \mathscr{S}} & =\iint K G\left(\theta ; \theta^{\prime}\right) \mathscr{S}\left(\theta^{\prime}\right) d \mathcal{A} d \mathcal{A}^{\prime}, \text { and } \\
F_{\mathscr{S} \mathscr{S}} & =\iint \mathscr{S}(\theta) G\left(\theta ; \theta^{\prime}\right) \mathscr{S}\left(\theta^{\prime}\right) d \mathcal{A} d \mathcal{A}^{\prime} .
\end{aligned}
$$

Substituting for $G\left(\theta ; \theta^{\prime}\right)$ and for $\mathscr{S}(\theta)$, and minimizing with respect to the angular positions $\omega_{i}$, we find that only the $F_{\mathscr{S} \mathscr{S}}$ term is important in determining $\omega_{i}$. After some algebraic manipulations, we find that the set of angular positions that minimizes the elastic free energy is given by

$$
2 \cos \omega_{i}=\frac{2 n-2 i+1}{n}
$$

and that the integrated Gaussian curvature between the symmetry-related walls at $\omega_{i}$ and $\left(\pi-\omega_{i}\right)$ is $2 \pi\left(2 \cos \omega_{i}\right)=$ $\frac{2 \pi}{n}(2 n-2 i+1)$. This directly leads to the result $(16)$ on the division of integrated Gaussian curvature.

\section{DISCUSSION AND CONCLUSIONS}

In this paper we have used a simplified version of the Ginzburg-Landau theory to predict the existence of stable topological wall defects (near the order-disorder transition) on spheres with $n$-atic order. Simulations and experiments can test our results. In our analysis we have ignored the effects of thermal fluctuations. Fluctuation effects will be important close to $T_{c}$. However, spheres are closed surfaces, and the system size is very small, thus diminishing the effects of fluctuations. The nature of order-disorder transition on orientationally ordered spheres is not clear and needs to be investigated. In particular, the transition may not be of the Kosterlitz-Thouless type (see, e.g., Ref. [13]). Close to $T_{c}$, interacting wall defects rather than point defects will dominate the transition. A detailed analysis of fluctuation effects is beyond the scope of this paper. In addition to fluctuation effects, it would be of interest to extend the theory to include the effects of anisotropy of elastic constants and study the shape changes of deformable vesicles, induced by wall defects. We have investigated the stability of topological wall defects on catenoids [27], which will be published elsewhere.

\section{ACKNOWLEDGMENTS}

We thank T. C. Lubensky and N. V. Madhusudana for useful discussions and Md. Arsalan Ashraf for help with graphics. Thanks are due to V. A. Raghunathan for many instructive discussions and to Joseph Samuel for helpful suggestions and a careful reading of the paper.
[1] See, for example, V. I. Arnold, Ordinary Differential Equations (Springer-Verlag, New York, 1992), Chap. 5; F. David in Statistical Mechanics of Membranes and Surfaces, edited by D. Nelson, T. Piran, and S. Weinberg (World Scientific, Singapore, 2004).

[2] T. C. Lubensky and J. Prost, J. Phys. II France 2, 371 (1992).

[3] J. Park, T. C. Lubensky, and F. C. MacKintosh, Europhys. Lett. 20, 279 (1992).

[4] D. R. Nelson, Nano Lett. 2, 1125 (2002).

[5] G. A. DeVries, M. Brunnbauer, Y. Hu, A. M. Jackson, B. Long, B. T. Neltner, O. Uzun, B. H. Wunsch, and F. Stellacci, Science 315, 358 (2007).

[6] T. Lopez-Leon, V. Koning, K. B. S. Devaiah, V. Vitelli, and A. Fernandez-Nieves, Nat. Phys. 7, 391 (2011), and references therein.
[7] V. Vitelli and D. R. Nelson, Phys. Rev. E 74, 021711 (2006).

[8] H. Shin, M. J. Bowick, and X. Xing, Phys. Rev. Lett. 101, 037802 (2008).

[9] X. Xing, H. Shin, M. J. Bowick, Z. Yao, L. Jia, and M.-H. Li, Proc. Natl. Acad. Sci. 109, 5202 (2012).

[10] L. Hirst, A. Ossowski, M. Fraser, J. Geng, J. Selinger, and R. L. B. Selinger, Proc. Natl. Acad. Sci. 110, 3242 (2013).

[11] O. V. Manyuhina and M. J. Bowick, Phys. Rev. Lett. 114, 117801 (2015).

[12] P. G. de Gennes and J. Prost, The Physics of Liquid Crystals (Clarendon Press, Oxford, 1993).

[13] P. M. Chaikin and T. C. Lubensky, Principles of Condensed Matter Physics (Cambridge Univ. Press, Cambridge, 1995).

[14] M. Kléman and O. D. Lavrentovich, Soft Matter Physics (Springer, New York, 2003). 
[15] P. Cladis and M. Kléman, J. Phys. (Paris) 33, 591 (1972).

[16] R. B. Meyer, Phil. Mag. 27, 405 (1973).

[17] For a review on continuous topological defects, see M. Kléman and J. Friedel, Rev. Mod. Phys. 80, 61 (2008).

[18] M. W. Deem and D. R. Nelson, Phys. Rev. E 53, 2551 (1996).

[19] J. M. Park and T. C. Lubensky, J. Phys. I France 6, 493 (1996).

[20] For a review, see M. J. Bowick and L. Giomi, Adv. Phys. 58, 449 (2009).

[21] $\mathscr{S}(\underline{\boldsymbol{\sigma}})=(2 \pi / \sqrt{g}) \sum_{m} q_{m} \delta^{(2)}\left(\underline{\boldsymbol{\sigma}}-\underline{\tilde{\boldsymbol{\sigma}}}_{m}\right)$ for isolated disclinations with point disclination charges $q_{m}$ located at $\tilde{\tilde{\sigma}}_{m}$. The charges $q_{m}$ have discrete values allowed by the $n$-atic orientational order.

[22] J.-P. Brasselet, J. Seade, and T. Suwa, Vector Fields on Singular Varieties (Springer, Heidelberg, 2009).
[23] Notice that $\oint_{0}^{2 \pi} d \alpha_{(\mathrm{lh})}-\oint_{0}^{2 \pi} d \alpha_{(\mathrm{uh})}=4 \pi$, where $\alpha_{(\mathrm{lh})}$ and $\alpha_{(\mathrm{uh})}$ refer to the lower and the upper hemisphere, respectively, with the integrals taken in the anticlockwise sense around the outward normal at the respective poles. Thus the index $4 \pi /(2 \pi)=$ 2 for the equatorial wall. This result is easily generalized to walls having other indices.

[24] As can be seen by putting $\theta=0$ in $\delta(\theta)=(1 /(2 \pi)) \int_{-\infty}^{\infty} e^{i k \theta} d k$ and setting the ultraviolet cutoff to $2 \pi / \zeta$.

[25] See L. Mesarec, W. Góźdź, A. Iglič, V. Kralj-Iglič, E. G. Virga, and S. Kralj, Sci. Rep. 9, 19742 (2019), and references therein.

[26] W. D. Luedtke and U. Landman, J. Phys. Chem. 100, 13323 (1996).

[27] C. Saichand, J. K. Alageshan, A. Roy, and Y. Hatwalne (unpublished). 